\title{
Frontières
}

\section{La mort qui vit dans la littérature d'enfance}

\section{Charlotte Guérette}

Volume 13, numéro 1, automne 2000

La mort au tableau noir

URI : https://id.erudit.org/iderudit/1074238ar

DOI : https://doi.org/10.7202/1074238ar

Aller au sommaire du numéro

Éditeur(s)

Université du Québec à Montréal

ISSN

1180-3479 (imprimé)

1916-0976 (numérique)

Découvrir la revue

Citer cet article

Guérette, C. (2000). La mort qui vit dans la littérature d'enfance. Frontières, 13(1), 6-11. https://doi.org/10.7202/1074238ar

\section{Résumé de l'article}

Que dire aux enfants concernant la mort, le deuil, la souffrance ? Faudrait-il se taire, feindre de les ignorer ou changer de sujet ? Nous proposons ici la littérature de jeunesse pour introduire ces thèmes très difficiles dans la classe. Dans les contes, les romans, les poèmes destinés à l'enfance, on en parle abondamment, simplement, justement. On en rit, on en pleure, on s'en moque... On en parle sincèrement! Quels livres choisir ? Comment les présenter? Ce sont de courtes, mais combien grandes questions auxquelles nous nous intéressons dans cet article. 


\section{Résumé}

Que dire aux enfants concernant la mort, le deuil, la souffrance ? Faudrait-il se taire, feindre de les ignorer ou changer de sujet? Nous proposons ici la littérature de jeunesse pour introduire ces thèmes très difficiles dans la classe. Dans les contes, les romans, les poèmes destinés à l'enfance, on en parle abondamment, simplement, justement. On en rit, on en pleure, on s'en moque... On en parle sincèrement! Quels livres choisir ? Comment les présenter ? Ce sont de courtes, mais combien grandes questions auxquelles nous nous intéressons dans cet article.

Mots clés : mort - littérature d'enfance - activités pédagogiques

\section{Abstract}

What should we say to children about death, mourning and suffering? When they ask about death, should we keep silent or change the topic? Here, we propose literature to introduce these very difficult themes in the classroom. Childhood tales, novels and poems can be used to broach this theme in a just yet simply way. One laughs, one cries, one mocks... but so sincerely! What book should one choose? How should it be presented? These important questions will be examined in this article.

Key words : children literature - pedagogical activities

\section{A R T I C L E}

\section{La mort qui vit dans la littérature d'enfance}

\section{" AUCUN VIVANT, AUCUN FANTÔME N'EST À L'ABRI. "}

\begin{abstract}
Charlotte Guérette,
professeure au Département d'etudes sur l'enseignement et l'apprentissage, Faculté des sciences de l'éducation de I'Université Laval.
\end{abstract}

Faut-il s'étonner que très peu d'études portent sur la manière d'aborder le thème de la mort et du deuil dans la classe ! Cette tâche prend facilement l'aspect d'un défi pour les enseignants. Pourtant, il y a à peine quelques décennies, l'école catholique québécoise donnait des réponses précises aux questions concernant la mort, le rite funéraire et l'au-delà. Dorénavant, dans une école passablement sécularisée, la réflexion sur le thème de la mort, dans une optique non confessionnelle, n'est guère privilégiée. D'une part, les futurs enseignants ne sont pas préparés à assumer cette tâche, d'autre part, les études sur la mort et le deuil en milieu scolaire sont grandement déficientes. Les futurs enseignants n'ont accès qu'à des connaissances partielles acquises au hasard des lectures. Qui parmi eux a étudié en profondeur les étapes du développement de l'enfant en relation avec la survenue de la mort dans leur vie ? Aussi, il est facile de vérifier que peu d'outils pédagogiques portant sur ce thème sont accessibles aux enseignants. Comment alors accompagner les enfants en quête de réponses sur les différentes manifestations de la mort, et de là, favoriser l'expression des perceptions, des réactions, des craintes qu'ils éprouvent face à elle?

À de nombreuses reprises dans ses travaux, Louis-Vincent Thomas a affirmé que l'homme a essayé de tuer la mort avec les armes du silence. Pour notre part, nous répondons à ce silence par la bouche des canons de la littérature d'enfance et de jeunesse, celle-là même où l'on rencontre la mort personnifiée. Nous croyons que cette littérature peut servir d'appui et d'accompagnement aux enfants qui cherchent à comprendre l'univers de la mort. Utilisés régulièrement et judicieusement, les contenus des récits qui développent ce thème contribuent largement à rendre significatifs, sinon à combler, les petits et les grands silences gravitant autour d'elle. Les héros et les intrigues d'ouvrages puisés à différents genres de la littérature d'enfance et de jeunesse vont nous en convaincre. Allons les rencontrer.

\section{DU MYTHE AU CONTE, LA MORT SE RACONTE}

De tout temps, le mystère de la mort a fasciné l'être humain. On se souvient du mythe d'Orphée qui descend aux Enfers pour chercher la belle Eurydice, morte de la morsure d'un serpent. Orphée, en fait, avait eu la permission des dieux de délivrer Eurydice de la mort. Par contre, son entreprise a échoué parce qu'il n'a pas respecté la condition qui consistait à ne pas regarder son amoureuse avant de franchir la porte des Enfers. Eurydice devait donc demeurer pour l'éternité avec le peuple des ombres.

Un nombre impressionnant de personnages mythiques ${ }^{1}$,aux attributs surnaturels, ont vaincu la mort, leur permettant ainsi de s'installer confortablement dans l'éternité. Qu'on pense à Gaïa, à Bacchus, à Apollon, à Athéna et à Hercule, nés aux confins du monde grec ancien. Ces dieux 
et déesses ont traversé le temps sans perdre tout à fait leur aura mythique.

L'homme moderne aimerait bien cesser de croire en ces héros et ces dieux immortels venus du monde d'autrefois. Certains ont même déserté leur propre religion pour se convertir aux nouvelles croyances de la science. Or, confrontés au vide et aux interrogations générées par la fin irréversible de la vie, ils cherchent encore quelques réponses pour calmer

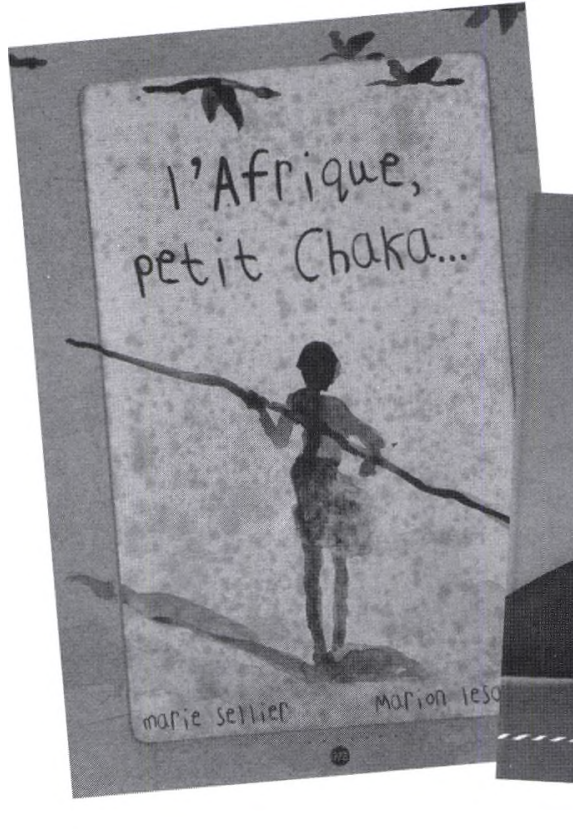

leurs angoisses. Les contes pour enfants, qui présentent des héros immortels, ne sont pas très différents des mythes. Ils reprennent, en les recomposant au goût du jour, les grands thèmes mythiques. Le goût du jour, dans le conte, produit un héros moins belliqueux, plus fraternel, moins monstrueux, plus convivial. Dans bon nombre de contes traditionnels ${ }^{2}$, la mort emporte celui qui ose s'en prendre au héros du récit; dans les contes modernes ${ }^{3}$, le méchant, c'est-à-dire celui qui s'attaque au héros, est épargné. Il sera même question, dans le conte moderne, de réconciliation. Pourtant, le conte n'ignore pas les scènes de mort. On y meurt comme on y naît, dans des conditions merveilleuses et incroyables. Bernadette Bricout ${ }^{4}$ précise que le conte entretient des relations complexes avec le mythe du fait que ses frontières mouvantes définissent les dimensions d'un univers multiforme où la mort se raconte invariablement.

\section{LA MORT DANS LA LITTÉRATURE D'ENFANCE ET LA MATURITÉ DES ENFANTS}

On remarque que les récits d'enfance qui abordent le thème de la mort portent en réalité sur celle des autres. À preuve, les quelques ouvrages proposés plus loin dans ce texte. Toutefois, il apparaît justifié de souligner, dès à présent, que si la mort concerne celle d'un autre, elle trouve néanmoins un écho dans l'inconscient de chaque enfant mis en présence de ce type de récit. À sa guise, et souvent à son insu, il en emporte les aspects vers sa maturité. Dennis Boyes ${ }^{5}$ souligne ce fait lorsqu'il affirme que les images implantées dans le psychisme, comme des semences, révèlent plus tard à la conscience leur sens profond. Ces récits imaginaires et chargés de symboles comptent parmi les artisans de la véritable identité de l'enfant. Ainsi, grâce à ces récits, le jeune arrive à mieux

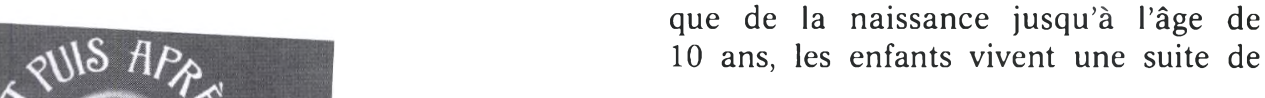

ses propres expériences de séparation, surtout celle, plutôt marquante pour le petit garçon, de la rupture de l'amour absolu de la mère. Pour sa part, la petite fille abandonne l'amour premier de sa mère pour réclamer celui de son père. S'ensuivent des abandons successifs qui sont autant d'étapes porteuses d'angoisse qui rappellent la fragilité mortelle de l'être humain. Pour Marie-Hélène EncrevéLambert $^{6}$, ces premiers "savoirs »sur la mort dépendent de ce que l'entourage a pu en dire et de l'expérience de l'enfant, mais surtout de la manière dont il vit sa relation affective avec ses parents, ses frères, ses sœurs et ses amis. Elle ajoute que de la naissance jusqu'à l'âge de

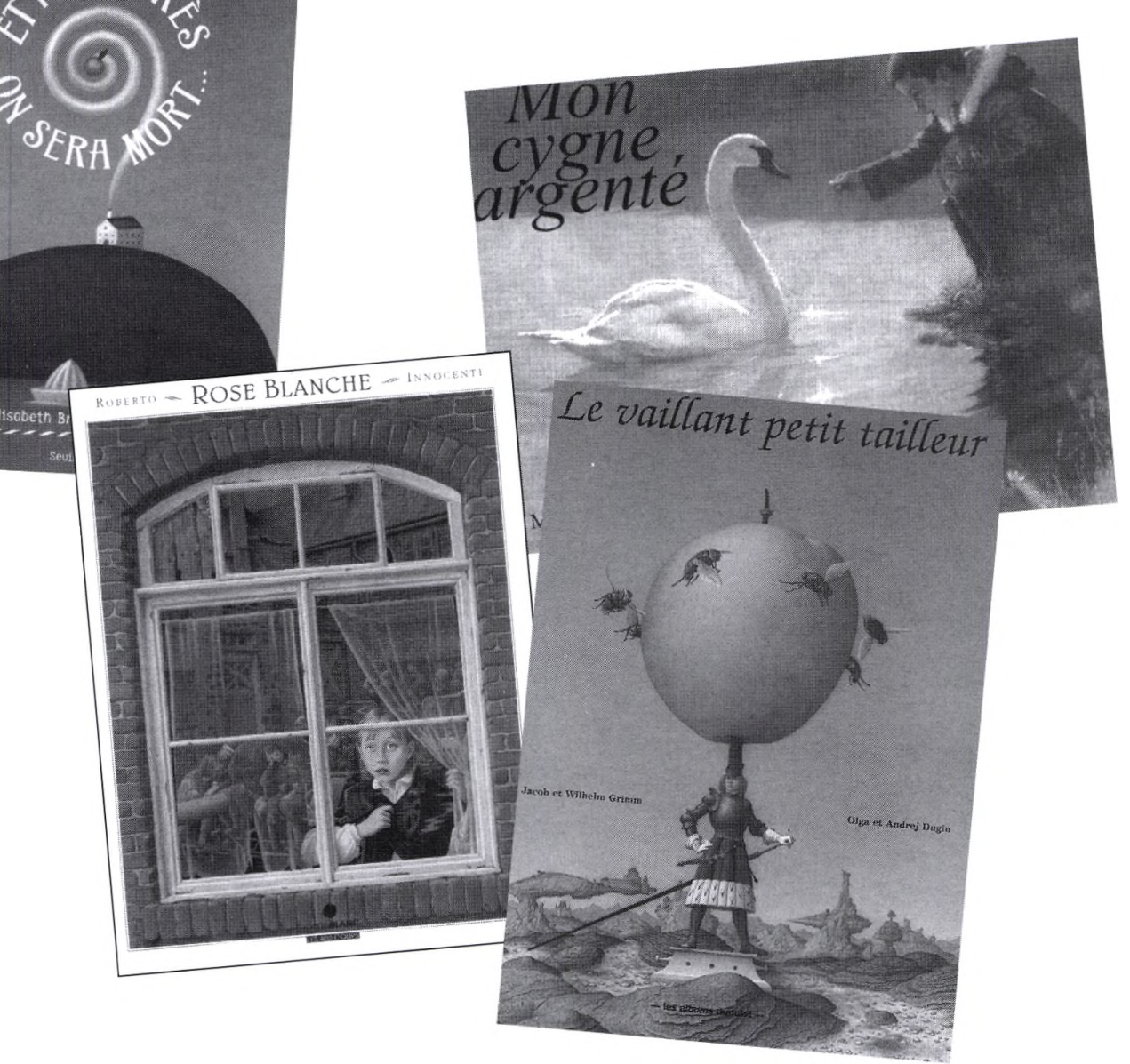

symboliser la mort, ce qui lui permet de mieux vivre ses propres peurs et ses propres angoisses. À cet égard, on sait que les plus grandes angoisses sont suscitées par un " défaut de représentation ». Un enfant qui n'arrive pas à se représenter la mort sera certes plus fragile.

\section{L'ANGOISSE DE LA SÉPARATION AU MENU DE PLUSIEURS CONTES}

Le thème de la séparation, et l'angoisse qui l'accompagne, se manifeste chez l'enfant dès qu'il commence à parler. Au fur et à mesure qu'il grandit, il prend conscience de la véritable signification de séparations douloureuses, mais nécessaires pour " grandir ». Il s'agit pour eux de créer de nouveaux liens affectifs avec leurs parents, ce qui leur permettra de partir à la rencontre des autres, du monde et du savoir, en ayant une bonne sécurité interne. À travers ces épreuves, les jeunes font l'expérience de ce qu'est la perte et le deuil.

Est-il besoin de rappeler que plusieurs contes de tradition orale font puissamment écho à ce thème ? Dans ces récits, les héros sont souvent forcés de vivre des séparations radicales avec leur famille. 
Dans L'anneau d'or', Le roi fait battre un ban et Le sabre de sept lieues et plusieurs autres titres, Tit-Jean, un célèbre héros de plusieurs contes québécois, quitte invariablement la maison familiale, sa région, ou encore son pays. Sa quête d'identité s'ouvre à des itinéraires divergents et suggère aux lecteurs bien autre chose que les figures modélisantes d'une socialisation étroite.

Le répertoire de contes traditionnels européens propose également plusieurs exemples directement associés au thème de la séparation. Parmi eux, Hansel et Gretel, ces deux enfants égarés volontairement en forêt par des parents trop pauvres pour les nourrir, Les trois petits cochons, forcés de fuir leur maison devant la ruse et l'appétit féroce d'un loup, et Blanche-Neige que sa mère veut tuer par excès de jalousie.

Alors que dans les titres cités précédemment, la séparation survient au début de l'intrigue, laissant au héros le temps de réparer les torts qui lui sont causés par cette situation, il est plus rare, mais possible, que celle-ci survienne à la fin du récit. Elle peut alors entraîner la mort d'un protagoniste. Ainsi, dans Les fées, le sort réservé à l'une des deux sœurs (la méchante) ne laisse planer aucune équivoque : "Elle se fit tant haïr que sa propre mère la chassa de chez elle ; et la malheureuse, après avoir bien couru sans trouver personne qui voulût la recevoir, alla mourir au coin d'un bois ».

À y regarder de près, on peut affirmer que tous les héros des contes sélectionnés ici sont porteurs d'anxiété et d'angoisse jamais exprimées ouvertement. On peut dire également qu'ils meurent symboliquement à certains aspects de leur vie. Ces passages obligés sont radicaux, sans possibilité de retour en arrière. On peut qualifier les différents épisodes de séparation de ces contes de premières morts symboliques devant préparer le héros à de nouvelles épreuves qui mettent en danger sa vie.

Il ne faudrait pas croire que cet aspect est négligé dans les contes modernes. Alors que la princesse, héroïne de $U n$ amour bon comme le sel est rejetée et chassée par un père orgueilleux et injuste, on note que, dans Le masque de brumes, c'est plutôt le héros qui s'impose une séparation volontaire alors qu'il part à la recherche d'un nouveau masque, symbole de la mémoire de son peuple. Parce qu'il ne sait rien faire d'autre que de dessiner des chats, le héros de L'enfant qui dessinait les chats est forcé de quitter sa famille, trop pauvre pour assurer sa subsistance. Une séparation à la fois douloureuse et annonciatrice de grandes réalisations.
Le conte s'avère une source première de l'expression du phénomène de la séparation, que François Roussel ${ }^{8}$ nomme mort/initiation ; séparation débouchant nécessairement sur le commencement d'une autre étape de vie. Une réalité récurrente du cheminement de plusieurs héros qui rejoint celle des lecteurs de ces récits. Comment pourrait-il en être autrement alors que la fonction symbolique ${ }^{9}$ du conte y trouve un lieu pour exprimer la séparation, la souffrance, l'épreuve, la mort ; un lieu aussi pour proposer aux enfants des solutions positives aux drames de leur propre vie. Des conclusions qui ouvrent sur l'espoir de vivre mieux sa vie d'enfant, celle d'avant la vraie mort.

\section{UN TEMPS POUR VIVRE, UN TEMPS POUR MOURIR}

À l'instar du mythe, le conte n'est pas situé dans le temps, ni dans l'espace réel. En ce sens, ces récits ne font pas ombrage à la réalité du jeune enfant qui ne maîtrise pas encore ces notions. Ce que met plutôt en lumière le contenu de chaque conte, c'est l'efficacité et la rapidité des actions qui animent le héros à travers l'intrigue du récit. En somme, la distance que le conte franchit de sa naissance à sa mort textuelle. Qu'il s'agisse du désormais célèbre chat dans le Chat botté, toujours à bout de souffle dans ses recherches pour trouver fortune pour son maître, faux marquis et pauvre comme Job, de la fille pauvre et mal-aimée dans Cendrillon qui a tout juste le temps de rentrer chez elle pour changer de vêtements entre deux bals, ou encore des trois petits cochons qui construisent des maisons à la rapidité de l'éclair dans Les trois petits cochons, le conte rappelle à l'enfant la relativité du temps qui passe et la brièveté de la vie d'un héros auquel il s'identifie. "Aussitôt dit, aussitôt fait ", dit le conte, alors que chacun sait pertinemment bien que pour mener ces actions à terme, les héros de ces contes auraient dû y consacrer des semaines, parfois même plusieurs années.

À l'opposé, la conclusion des contes de fées prend souvent une saveur d'éternité. Se peut-il que les protagonistes des contes vivent heureux pour toujours ? Si telle est la vérité du conte, l'enfant ne peut croire qu'ils puissent vivre éternellement. D'autre part, la transformation vécue par chacun des héros entre le début et la fin du conte ne suggère-t-elle pas à l'enfant que celle-ci puisse se poursuivre, le conte terminé ? Donc, qu'il peut exister un " après-conte » que personne n'a jamais cru bon de revenir nous raconter. Si le non-dit du conte est un hommage à la vie, il n'exclut pas la présence de la mort.

\section{LA RENCONTRE AVEC LA MORT ET LE DEUIL}

Chacun sait que la rencontre de l'enfant avec la mort, sous une forme ou sous une autre, est normale et inévitable. C'est généralement vers l'âge de trois ans que le jeune se rend compte qu'elle peut arriver à quelqu'un, mais pas à lui. Qu'il soit témoin de la maladie et du décès de son animal préféré, qu'il vive celle d'un ami, d'un camarade de classe, d'un parent cher, il ne comprend toutefois pas que la mort est un élément de la vie (la plupart des enfants y parviennent vers l'âge de 10 ans).

Vers l'âge de sept ans, les enfants commencent à comprendre que la mort est irréversible. Certains parmi eux ne le réaliseront que des années plus tard. Léana a environ 11 ans dans le roman Le signe de l'albatros. Elle exprime clairement ce fait quand elle supplie Chico, le capitaine de L'Espéranza, gravement blessé dans une tempête en mer, de lui dire s'il est mort.

Ici, on retiendra que plusieurs contenus de contes modernes et de romans peuvent être utilisés dans une perspective de support au partage et à la compréhension des différents aspects relevés plus haut. Ainsi, au fur et à mesure de la lecture de Et puis après, on sera mort..., le jeune lecteur peut vérifier que : "Comme tout ce qui est né, comme tout ce qui est vivant, un jour, il finira de vivre. Après, il sera mort. Et alors ? Et après ? Ça, personne ne le sait ". Lalbum $A u$ revoir grand-père relate la dernière visite d'un petit-fils à son grand-papa qui mourra bientôt. Le thème de la mort d'un être cher y est abordé avec réalisme et délicatesse. Le contenu de Les chevaux des nuages exprime à la fois le chagrin de deux jeunes sœurs orphelines et les doux souvenirs qu'elles gardent de leur mère. Le loup rouge, un conte moderne d'animaux décrit plusieurs moments de la vie d'un chien adopté. À la fin de l'ouvrage, les principaux épisodes du récit sont repris sous la forme de vignettes d'une bande dessinée : des souvenirs de la vie du héros mis en images. Un autre conte d'animaux, Pochée, décrit l'accident mortel qui sépare deux tortues, et comment Pochée, la tortue survivante vit son deuil. Dans Rose Blanche, un album à portée réaliste, le lecteur découvre des épisodes de la vie d'une fillette qui vécut dans un village allemand pendant la guerre. Un jour, elle disparut. Nul ne la revit jamais ! Dans Feng, fils $d u$ vent, un vieux sage apprend au jeune Feng l'art de faire voler un cerf-volant. Sa mission accomplie, il meurt. Dans l'avant-propos de Mon cygne argenté, un récit qui met en lumière l'amitié profonde d'un jeune garçon et d'une 
mère-cygne vouée à un destin tragique, Orlando Gibbon décrit ainsi la rencontre du cygne avec la mort :

Le cygne argenté jamais ne chante

Mais qu'approche la mort et dans

sa roselière

Il incline la tête et sa voix nous

enchante.

Pour la première fois mais aussi la dernière,

Il chante, puis il se tait, à jamais.

Enfin, suggérant que si la fin définitive d'un héros est une évidence et du même souffle qu'il est quand même possible de réaliser certains de ses désirs à ce moment, Vieux Thomas dans Vieux Thomas et la petite fée le révèle ainsi au lecteur : "Sachant qu'il allait mourir, il se leva péniblement et marcha lentement vers la mer. C'est là qu'il souhaitait disparaître ".

Dans la production récente de romans pour l'enfance, l'avant-mort et l'aprèsmort d'une personne chère sont aussi abordés. À titre d'exemples, nous retiendrons ici quelques titres de romans à portée réaliste qui sont caractéristiques du souci de leurs auteurs de présenter différentes situations associées à la mort. Ainsi, dans Un grand-père tombé du ciel, Leah apprend à connaître son grand-père dont elle a longtemps ignoré l'existence. Elle l'accompagne dans les derniers moments de sa vie. Les événements entourant la maladie, le décès et les obsèques d'une grand-mère sont décrits avec justesse dans Adieu, mamie; le grand-père de Faustine l'accompagne dans une démarche sur le deuil et le souvenir dans Faustine et le souvenir, un classique de la littérature d'enfance.

\section{LES PEURS ET LA MORT DANS LES RÉCITS D'ENFANCE}

La majorité des enfants présentent, en ce qui a trait à la peur, les caractéristiques suivantes : ils ont tendance à avoir peur des animaux, du noir, des créatures imaginaires et d'être séparés des parents. Par la suite, ils ont peur des situations qui sont liées aux fondements même de leur existence dont celle de la mort. Bon nombre de livres de contes pour enfants exploitent des peurs réelles ou imaginaires ; plusieurs exemples d'ouvrages proposés dans les différentes parties de ce texte tendent à le démontrer. Ce faisant, ils peuvent être objets de stimulation de leur imagination et de leur intelligence. Ils peuvent aussi contribuer à clarifier les émotions des enfants.

Parce que les contes disposent d'un atout notable, la fiction, on leur accorde le droit d'exprimer ou de suggérer la présence de la mort. C'est sur elle que s'ap- puient les actions des protagonistes de plusieurs récits. L'enjeu de vie et de mort y est donc manifeste ou fortement suggéré, illustrant ainsi plusieurs des peurs qui peuvent émerger au cours du développement affectif de l'enfant. Qu'il suffise de citer l'exemple des Contes des Mille et Une nuits dont s'inspirent, souvent à leur insu, de nombreux contes de tradition orale et des contes modernes.

\section{LES PERSONNAGES ASSOCIÉS À LA MORT DANS LA LITTÉRATURE D'ENFANCE}

Claude de la Genardière ${ }^{10}$ souligne que les personnages des contes sont des figures du moi, le moi du lecteur, en l'occurrence, divisé par ses contradictions et ses combats intimes, mais qui aspire à une paix, pareille à celle du héros au terme du récit.

Imbriquée à la trame des épisodes de plusieurs œuvres littéraires, la mort peut y revêtir la forme de personnages caractéristiques : loup-garou, ogre, géant, sorcière, mauvaise fée, méchant magicien, dragon, troll, kobold et autres membres de la grande famille des créatures maléfiques, ces personnages qui symbolisent la mort et par le fait même, les limites de la condition humaine. Néanmoins, on constate que la majorité des personnages, réels et imaginaires, humains et animaux, identifiés dans le corpus des ouvrages d'enfance, peuvent véhiculer le thème de la mort aussi bien par leur côté obscur que par leur côté lumineux, aussi bien par l'horreur que par l'humour. Il s'agit là du choix d'un auteur souvent inspiré de ses lectures antérieures, de ses expériences personnelles et de sa connaissance du développement affectif des jeunes. Nous présenterons plus en détail quelques-unes de ces figures, compte tenu de leur présence dans les contenus d'un grand nombre d'ouvrages d'enfance publiés récemment.

\section{LES ÊTRES HUMAINS}

Une représentation récurrente des formes humaines qu'a pu prendre la mort au cours des siècles concerne une femme à l'allure mystérieuse, vêtue de noir, à la situation sociale mal définie, à la silhouette étrange, qui n'offre d'autre alternative au héros que la rupture définitive avec la vie. L'exemple du grand classique $L a$ mort-marraine démontre qu'une femme, à elle seule, contrôle le destin (vie et mort) de tous les êtres humains. C'est elle qui décide de laisser brûler ou d'éteindre la bougie allumée pour chaque homme, femme et enfant, ce qui l'investit d'un pouvoir exceptionnel. Au contraire, dans Bonjour, Madame la Mort, un conte moderne publié récemment, la mort prend les traits d'une femme sympathique et incite une paysanne centenaire à la suivre dans l'au-delà.

La mort symbolisée par une femme n'a toutefois pas toujours le dernier mot dans les contes. Ainsi, Madame Misère, un récit inspiré d'une légende portugaise, fait la preuve que les pouvoirs magiques de cette vieille dame lui permirent d'exiger de la mort qu'elle la laisse vivre éternellement. Malgré cela, aucun lecteur de ce récit n'enviera son sort !

Dans plusieurs contes de tradition orale, les rois imposent des épreuves aux prétendants qui demandent la main de leur fille. À ces occasions, le héros se place volontairement en danger de mort. Ainsi, pour épouser une princesse, le petit tailleur du conte Le vaillant petit tailleur doit affronter des obstacles titanesques : tuer deux géants, capturer une licorne et faire prisonnier un cruel sanglier. Dans La montagne d'Ivoire, Tit-Jean répond à l'annonce d'un roi résigné à donner la moitié de sa fortune et sa fille captive à qui la délivrerait de la montagne d'Ivoire. Mais pour y parvenir, Tit-Jean doit affronter un déluge, un dragon et un sorcier. Peu importe finalement l'ampleur des tâches à accomplir et des dangers qu'elles comportent, le héros y parviendra. De précieuses victoires de la vie sur la mort !

\section{LE LOUP, UN ANIMAL POPULAIRE DANS LES CONTES}

L'animal qui a le plus souvent véhiculé la peur et la mort dans les contes est sans conteste le loup. Le grand méchant loup des contes rôde souvent dans la forêt prêt à croquer sa proie. Il est par excellence La bête, l'hôte malfaisant et cruel de la forêt ${ }^{11}$. Il concrétise les dangers de ce lieu mystérieux. À cela, Claude de la Genardière ${ }^{12}$ ajoute que parce qu'il existe, on peut raconter à son sujet des histoires qui sont vraies, des histoires exemplaires empreintes de morale, et celles qu'on associe à la rumeur. Le petit chaperon rouge, comme la grande majorité des contes de tradition orale, appartient à la catégorie des histoires exemplaires qui visent à prévenir l'enfant du danger de mort.

Ici encore, des distinctions nettes et récurrentes apparaissent lorsqu'il s'agit d'identifier les caractéristiques et le rôle du loup dans le conte moderne. Il ne s'agit plus de le présenter comme un dévoreur d'enfants, mais plutôt de se moquer de lui ou de s'en faire un ami, parfois aussi de le vaincre. Parmi les nombreux exemples qu'on pourrait relever, nous avons retenu les suivants : $L e$ loup, mon oeil où une petite fille cochon tourne en dérision un loup qui voulait la 
manger dans sa soupe, Le jeune loup qui n'avait pas de nom qui développe le thème de la recherche d'identité d'un jeune loup, septième de sa famille, et Goliath et le loup, le récit d'un enfant qui dévore un loup, plutôt que d'être croqué par lui.

\section{LES ÊTRES SURNATURELS}

Déjà présents dans la mythologie, les sorcières, les dragons, les ogres et les géants retiennent l'attention lorsqu'il s'agit de faire une association directe entre ces personnages, leur présence dans le corpus d'œuvres d'enfance et le thème de la mort.

Il fut un temps où le pouvoir de la sorcière $^{13}$ était total. Dans les sociétés de type traditionnel, elle présidait à la vie et à la mort, veillait aux récoltes, contrôlait les éléments et les hommes. Puis on en vient à prétendre qu'elle jette des sorts. Elle incarne le mal, la laideur. On l'associe à la nuit, aux espaces inquiétants. Progressivement, elle devient le noyau, le centre de tout ce qu'on ne peut comprendre et admettre chez les femmes.

Rarement identifiée au personnage principal des contes, la sorcière a toutefois un pouvoir immense sur leur déroulement. Qu'il suffise de souligner ses intentions meurtrières dans Hansel et Gretel, du sort qu'elle réserve au fils du tsar dans La princesse grenouille et de tous les autres gestes destructeurs qu'on lui attribue.

Selon Gaborit et Guesdon ${ }^{4}$, le personnage de la sorcière a glissé pour devenir dans plusieurs contes modernes un personnage stéréotypé, exsangue, souvent humoristique. Il faut alors réunir plusieurs personnages de sorcières pour tenter d'y retrouver la complétude du mythe premier. Dans ces contes, on tente de dédramatiser la peur de la mort qu'elle pourrait générer. Par exemple, dans $L a$ sorcière de la rue Mouffetard, l'auteur raconte les péripéties drolatiques d'une sorcière vivant à Paris, Wittilda, drôle de sorcière qui a 47 chats, pas beaucoup de sous, et qui décide de trouver du travail. Une sorcière qui jette un sort au roi dans L'étoffe d'un roi n'a d'autre alternative que de prendre ses jambes à son cou, à la fin du conte. Ce sont là quelques exemples qui tendent à montrer que pour identifier le mythe initial dans les contes modernes, il faut nécessairement sonder le texte pour retrouver la sorcière valorisée, puissante, portant avec elle son passé de femme totale et provocante. Il est permis de douter que tous les enfants y parviennent seuls !

De dragon à ogre et à géant, il n'y a qu'un pas puisque ces êtres surnaturels sont associés aux forces du mal dans les contes de tradition orale et les légendes. Ils représentent le plus souvent ce qui subsiste dans l'homme de pulsions d'agressivité. Tueurs par nature, ils ont la force et le pouvoir de détruire les héros qu'ils rencontrent sur leur route. Les vaincre signifie une victoire sur la mort Bien que ces caractéristiques s'appliquent globalement à ces trois êtres surnaturels, il importe d'apporter quelques distinctions particulières en ce qui concerne les ogres. Ce monstre apparaît souvent comme un géant vivant au plus profond de la forêt ; il est riche et possède des objets magiques. Il porte en lui l'opposition vie/mort; par conséquent, il peut faire mourir ou sauver de la mort. Celui que rencontre Le chat botté en possède toutes les caractéristiques. Mais l'ogre peut aussi être représenté sous des formes quelque peu différentes. Par exemple, le grand personnage rouge qui entraîne les enfants comme des rats dans Le joueur de flûte de Hamelin, la belle-mère de Blanche Neige qui veut non seulement la tuer mais aussi manger son foie et ses poumons, et Barbe-bleue, figure d'un mari abusif s'arrogeant un pouvoir absolu sur son "épouse-esclave » dont il dévore la personnalité. La véritable nature de l'ogre révélée !

On remarque que les contes modernes tendent à véhiculer une image souvent opposée de ce personnage. Dans L'ogre et la bête inconnue, deux paysans parviennent à noyer un ogre, leur seigneur, alors que ce monstre devient cuisinier dans $L e$ monstre-Mange-Bébés.

\section{L'UTILISATION DES LIVRES QUI ABORDENT LE THĖME DE LA MORT EN CLASSE DU PRIMAIRE}

Aucun adulte œuvrant auprès des enfants ne peut négliger d'accorder une attention soutenue au thème de la mort dans la littérature d'enfance. EncrevéLambert ${ }^{15}$ souligne que les livres qui l'abordent permettent de dialoguer avec les enfants sur la perte, la fin des choses, la séparation et la mort, à travers la lecture faite ensemble, en dehors de toute expérience de deuil.

Il n'en demeure pas moins que plusieurs adultes se sentent démunis pour accompagner efficacement, à ces occasions, les jeunes. Nous avons voulu mettre en évidence la présence significative du thème de la mort dans la littérature d'enfance et dans le développement des enfants. Il nous apparaît aussi approprié de proposer quelques pistes devant favoriser l'utilisation de ces ouvrages en milieu scolaire. Celles-ci concernent les activités d'animation de la lecture : récit, lecture, activités d'échange et de discussion. Autant de pratiques éducatives que l'enseignant peut réaliser dans une perspective de support à la compréhension et à l'expression des perceptions et des réactions des jeunes devant les différents visages que revêt la mort en littérature d'enfance.

Chacun peut trouver, dans l'univers fictif de la littérature d'enfance, des réponses aux questions qu'il se pose. Bruno de la Salle attribue une valeur inestimable au récit et à la lecture de contes lorsqu'il affirme : "Si vous désirez vraiment laisser vivre votre conte de façon profitable, regardez dehors, acceptez l'idée de vous retrouver à côté d'un monstre réel qui pourrait vous manger ou vous écraser!16 ". Pour laisser vivre un conte qui aborde le thème de la mort, il est primordial que l'adulte effectue d'abord une rencontre personnelle avec son contenu. C'est donc dire qu'il lui sera souvent nécessaire de le lire plusieurs fois, silencieusement et à voix haute, pour fixer dans sa mémoire les traits principaux du texte. Une étape cruciale et déterminante de la réussite de ce type d'activité : s'approprier les récits et apprendre à se découvrir à travers eux !

Il va sans dire que cette préparation s'applique aussi aux romans, à quelques variantes près. Ainsi, on peut opter pour la lecture complète de l'ouvrage, celle de certains passages, aussi bien que pour le récit-synthèse des épisodes qui sont moins importants. Dans tous ces cas, on peut prévoir que l'utilisation d'un seul titre occupera plusieurs séances de lecture.

Suite au récit ou à la lecture d'un ouvrage qui aborde le thème de la mort, il est nécessaire d'en parler avec les enfants. Encrevé-Lambert ${ }^{\not}$ précise qu'il s'agit moins d'en parler, que de les entendre nous en parler. Alors comment recevoir leurs questionnements, comment accueillir leurs découvertes, comment accompagner leurs angoisses ? Les ouvrages pour enfants qui abordent le thème de la mort sont porteurs de mots et de réalités sur lesquels l'adulte peut appuyer sa propre parole pour offrir aux jeunes un lieu d'échange où leur parole, celle du livre et celle de l'adulte se rejoignent et se complètent. Un bref dialogue puisé au conte initiatique L'Afrique, petit Chaka illustre bien cette possibilité : "Moi, je ne veux pas que tu meures, jamais ! dit petit Chaka à son grand père ». Celui-ci répondit : " La mort est un vêtement que tout le monde portera ". Et qu'ajouteront à leur tour les enfants et l'adulte? 


\section{CONCLUSION}

Refuser de jeter un regard attentif à la mort qui vit dans la littérature d'enfance ne fait qu'obscurcir le voile dont on l'a entourée trop souvent et trop longtemps. Les ouvrages qui abordent cette question sont porteurs de vérités qui contribuent à en humaniser les différents aspects. Des vérités dont la signification se manifeste alors qu'on choisit d'en parler encore et encore. Comme il a été permis de le vérifier à plusieurs occasions, des ouvrages puisés au répertoire des récits de tradition orale proposent aux enfants des visages de la mort différents de ceux identifiés dans la littérature dite moderne. Il ne s'agit peut-être que de permettre à ces jeunes de parvenir, un jour, à la regarder bien en face et à en parler ${ }^{18}$. En parler tout simplement ! Parler de la mort avec les enfants, n'est-ce pas d'abord et avant tout parler de la vie ?

\section{Notes}

1 On peut trouver la liste détaillée des personnages en cause ici dans Colette EPSTIN et Hélène LAPORTE, Le livre de la mythologie grecque et romaine, Paris, Gallimard, collection Découverte Cadet, 1999.

2 En littérature d'enfance, on utilise une classification des contes selon leur origine et leur contenu. L'origine du conte de tradition orale se perd dans l'histoire de l'humanité. C'est un récit de fiction, il est anonyme. Il n'est pas daté et fait partie du folklore.

3 Par conte moderne, on entend généralement ceux écrits par des auteurs connus depuis le milieu du XIXe siècle.

4 Cette partie de texte s'inspire de Bernadette BRICOUT, "Conte et mythe " dans P. BRUNEL (dir. publ.), Dictionnaire des mythes littéraires, Paris, Du Rocher, 1988 , p. 368.

5 Dennis BOYES, Initiation et sagesse des contes de fées, Paris, Albin Michel, 1988, p. $87-88$

6 Marie-Hélène ENCREVÉ-LAMBERT, La mort, Paris, Bayard, 1999, p. 37.

7 La bibliographie des ouvrages d'enfance cités est proposée à la fin du texte.

8 François ROUSSEL, Les contes de fées: lecture initiatique, Plazac-Rouffignac, Amrita, 1993, p. 139.

9 De nombreux spécialistes du conte et de la psychanalyse attribuent une importance primordiale à la fonction symbolique dans les contes. C'est donc dire que ces récits ont une double signification : celle que dit le texte et qui à son tour trouve sa signification profonde alors qu'il rejoint l'inconscient du lecteur ou de l'auditeur de ces récits.

10 Claude DE LA GENARDIÈRE, Encore un conte ? Le Petit Chaperon Rouge à l'usage des adultes, Nancy, Presses universitaires de Nancy, 1993, p. 9.

11 René-Lucien ROUSSEAU, L'envers des contes, Saint-Jean-de-Braye, Dangles, 1988 , p. 65
12 Claude DE LA GENARDIÈRE, ibid. note 10, p. 125.

13 Lydia GABORIT; Yveline GUESDON et AL. "Les sorcières " dans Dictionnaire des mythes littéraires, p. 1307.

14 Ibid., p. 1319.

15 Marie-Hélène ENCREVÉ-LAMBERT, ibid. note 6, p. 133.

16 Bruno DE LA SALLE, Le Conteur amoureux, Tournai, Casterman, 1995, p. 97.

15 Marie-Hélène ENCREVÉ-LAMBERT, ibid. note 6, p. 14

18 Brigitte LABBÉ et Michel PUECH, La vie et la mort, Toulouse, Milan, 2000, p. 38.

\section{BIBLIOGRAPHIE D'OUVRAGES D'ENFANCE CITÉS}

"L'Ogre et la bête inconnue " dans Contes d'Ogres et de Sorcières, Ill, de Christian HEINRICH. Toulouse, Milan, 1999, p. 73-79.

BARBEAU, Marius, "L'anneau d'or » dans Morvette et Poisson d'or, Montréal, Hurtubise $\mathrm{HMH}, 2000$, p. 80-90.

BARBEAU, Marius, "La montagne d'Ivoire " dans Morvette et Poisson d'Or. Montréal, Hurtubise $\mathrm{HMH}$, p. 34-41.

BARBEAU, Marius, "Le roi fait battre un ban " dans Morvette et Poisson d'or, Montréal, Hurtubise $\mathrm{HMH}, 2000$, p. 51-63. BARBEAU, Marius. "Le sabre de sept lieues " dans L'Oiseau d'Eurémus, Montréal, Hurtubise $\mathrm{HMH}, 2000$, p. 121 129.

BEAUDE, Pierre-Marie, Le signe de l'Albatros, Paris, Castor Poche Flammarion, 1994

BRAMI, Élisabeth et SCHAMP, Tom, Et puis après, on sera mort... Paris, Seuil Jeunesse, 2000, p. 18-23

BUEIHNER, Caralyn et Mark, Wittilda, drôle de sorcière, Paris, Bayard, 1999

CHAPEAU, Thierry L'étoffe d'un roi, Strasbourg, Callicéphale, 2000.

CLÉMENT, Claude, Le masque de brumes, Toulouse, Éditions Milan, 1998.

COJAN-NEGULESCO, Mariane, Un amour bon comme le sel, Paris, Albin Michel Jeunesse, 1998.

DDEDIEU, Thierry, Feng, fils du vent, Paris, Seuil Jeunesse, 1995.

DEMERS, Dominique, Vieux Thomas et la petite fée, Saint-Lambert, Dominique et Compagnie, 2000, p. 28.

GALLAS, Christophe et INNOCENTI, Robert, Rose Blanche, Montréal, Les 400 coups, 1999

GRAVEL, François, Madame Misère, Montréal, Les 400 coups, 2000.

GRIMM, Jacob et Wilhelm (d'après), Hansel et Gretel, Paris, Gründ, 1998.

GRIMM, Jacob et Wilhelm, "BlancheNeige " dans Les grands contes de Grimm, Paris, Albin Michel Jeunesse, 1999, p. 7187.

GRIMM, Jacob et Wilhelm, " Le petit chaperon rouge " dans Les grands contes de Grimm, Paris, Albin Michel Jeunesse, 1999, p. $159-167$

GRIMM, Jacob et Wilhelm, Le vaillant petit tailleur, Tournai, Casterman, 1999.

GRIPARI, Pierre, "La sorcière de la rue Mouffetard " dans La sorcière de la rue Mouffetard et autres contes de la rue Broca, Paris, Gallimard Jeunesse, 1997.
HASSAN, Yaël, Un grand-père tombé $d u$ ciel, Tournai, Casterman, 1997.

LAMOTHE, Raymonde, Adieu, mamie, Montréal, Pierre Tisseyre, 2000.

LAPORTE, Michel, 10 contes des Mille et Une nuits, Paris, Castor Poche Flammarion, 2000.

LEAVY, Una, Au revoir Grand-Père, Paris, Bayard, 1996.

LEMIEUX, Michèle, Le joueur de flûte d'Hamelin, Fribourg, Calligram, 1996.

Les trois petits cochons, Paris, Nathan, 1997.

LEVINE, Arthur, L'enfant qui dessinait les chats, Peintures de Frédéric Clément, Paris, Pastel/L'école des loisirs, 1993.

LEWIS, J. Patrick, La princesse grenouille, un conte russe, Tournai, Casterman, 1997. MEDDAUGH, Susan, Le loup, mon ail, Paris, Autrement Jeunesse, 1998.

MORPURGO, Michael, Mon cygne argenté, Paris, Pastel/L'école des loisirs, 2000, p. 2 .

MOURLEVAT, Jean-Claude, Le jeune loup qui n'avait pas de nom, Toulouse, Milan, 1998.

PERNUSCH, Sandrine, Faustine et le souvenir, Tournai, Casterman, 1998

PERRAULT, Charles, "La Barbe-bleue " dans Contes de ma Mère l'Oye, Paris, Gallimard, 1998, p. 47-59.

PERRAUlT, Charles, Cendrillon, Zurich, Nord-Sud, 1999.

PERRAULT, Charles, Le chat botté, Zurich, Nord-Sud, 1999.

PERRAULT, Charles, Les fées, Paris, L'école des loisirs, 2000, p. 40.

QUESMAND, Anne, La mort-marraine, Moulins, Ipomée, 1990.

RICHÉ, Nathalie, "Le monstre MangeBébés " dans Les plus beaux contes de Toboggan, Toulouse, Milan, 1999, p. 74-80. ROSENBERG, Liz, Les chevaux des nuages, Paris, Bayard, 1997.

SELLIER, Marie, L'Afrique, petit Chaka, Paris, Réunion des Musée nationaux, 2000. SEYVOS, Florence, Pochée, Paris, L'école des loisirs, 1994.

TEUlADE, Pascal, Bonjour, Madame la Mort, Paris, L'école des loisirs, 1997.

WAECHTER, F. K., Le loup rouge, Paris, L'école des loisirs, 1998. 